\title{
ON THE NUMBER OF ZEROS OF DIAGONAL FORMS
}

\author{
TSUNEO ISHIKAWA
}

\begin{abstract}
In this paper, we study the number of zeros of the equation $X_{1}^{e}+$ $X_{2}^{e}+\cdots+X_{n}^{e} \equiv 0(\bmod p)$, where $e>1$ is a positive integer and $p \equiv 1$ $(\bmod e)$ is a prime. For $e=5,7$, we compute the recurrence satisfied by these numbers, using the generalized Jacobi sums.
\end{abstract}

\section{INTRODUCTION}

Let $e>1$ be a positive integer and let $p$ be a prime, $p \equiv 1(\bmod e)$. We consider the number of solutions of the equation

$$
X_{1}^{e}+X_{2}^{e}+\cdots+X_{n}^{e}=0
$$

in the finite field $\mathbb{F}_{p}$. Let $T_{e}(n)$ denote the number of such solutions. In the case of $e=3$, Chowla, Cowles, and Cowles proved in [3], using Gauss sums, that for $p \equiv 1(\bmod 3)$

$$
\sum_{n=1}^{\infty} T_{3}(n) x^{n}=\frac{x}{1-p x}+\frac{x^{2}(p-1)(2+d x)}{1-3 p x^{2}-p d x^{3}}
$$

where $d$ is uniquely determined by

$$
4 p=d^{2}+27 b^{2} \text { and } d \equiv 1 \quad(\bmod 3) .
$$

Myerson settled the case of $e=4$ in the same manner in [11]. It is easy to do the cases $e=3,4$ and 6 , because the associated Gauss sum relates to the imaginary quadratic field. In [12], Richman gives a formula for the generating function in terms of the period polynomial of the Gauss sums, but it is difficult to find a formula for this polynomial.

In this paper, we shall consider an algorithm to obtain the recurrence for $T_{e}(n)$, using generalized Jacobi sums and certain quadratic partitions of $p$ in some cases. But we cannot express the recurrence in general form, because the partition is very intricate except when $e=3,4,6$ and 8 . For $e=5$ and 7, if $p<1000$, we give the recurrence explicitly in Tables 2 and 5, and they may suggest some interesting conjectures. Our method can be applied in the case that the usual Jacobi sums or the cyclotomic numbers of order $e$ are known explicitly,

Received by the editor September 23, 1991 and, in revised form, March 27, 1992, March 30, 1993, and April 26, 1994.

1991 Mathematics Subject Classification. Primary 11D79, 11T22, 11 T23. 
that is, $e=2,3,4,5,6,7,8,9,10,11,12,14,15,16,18,20,24$ and 30 (see $[1,2,4,6-10,13,14])$.

\section{RECURRENCE}

In this section, we show how to obtain the recurrence for $T_{e}(n)$, using generalized Jacobi sums. The fact that the $T_{e}(n)$ satisfy a recurrence was already proved by Chowla et al. in [3] and Richman in [12].

Now, since

$$
\sum_{t=0}^{p-1} \zeta_{p}{ }^{t a}= \begin{cases}p & \text { if } a \equiv 0(\bmod p) \\ 0 & \text { otherwise }\end{cases}
$$

where $\zeta_{p}=\exp \left(\frac{2 \pi i}{p}\right)$, we have

$$
p T_{e}(n)=\sum_{t=0}^{p-1} \sum_{x_{1}=0}^{p-1} \sum_{x_{2}=0}^{p-1} \cdots \sum_{x_{n}=0}^{p-1} \zeta_{p}^{t\left(x_{1}^{e}+x_{2}^{e}+\cdots+x_{n}^{e}\right)}=\sum_{t=0}^{p-1}\left(\sum_{x_{1}=0}^{p-1} \zeta_{p}^{t x^{e}}\right)^{n}=\sum_{t=0}^{p-1} G(t)^{n}
$$

where $G(t)$ is the Gauss sum of order $e$ :

$$
G(t)=\sum_{x_{1}=0}^{p-1} \zeta_{p}^{t x^{e}}
$$

We take $\Delta$ in $\mathbb{F}_{p}$ such that $\Delta^{d}$ is not an $e$ th power for any $d(0<d<e)$. Then, the multiplicative group $\mathbb{F}_{p}^{*}$ of $\mathbb{F}_{p}$ has the following coset decomposition:

$$
\mathbb{F}_{p}^{*}=\left(\mathbb{F}_{p}^{*}\right)^{e}+\Delta\left(\mathbb{F}_{p}^{*}\right)^{e}+\Delta^{2}\left(\mathbb{F}_{p}^{*}\right)^{e}+\cdots+\Delta^{e-1}\left(\mathbb{F}_{p}^{*}\right)^{e} .
$$

Notice that each coset has $\frac{p-1}{e}$ elements and that $G(u)=G(v)$ for any $u, v \in$ $\Delta^{j}\left(\mathbb{F}_{p}^{*}\right)^{e}(j=0,1, \ldots, e-1)$. Since $G(0)=p$, we have

$$
p T_{e}(n)=\frac{p-1}{e} \sum_{j=0}^{e-1} G\left(\Delta^{j}\right)^{n}+p^{n} .
$$

Thus, $G\left(\Delta^{j}\right)(j=0,1, \ldots, e-1)$ are roots of the polynomial

$$
F_{e}(x)=x^{e}+y_{1} x^{e-1}+y_{2} x^{e-2}+\cdots+y_{e},
$$

where $y_{i}=-\frac{1}{i} \sum_{j=0}^{i-1} y_{j} s_{i-j}$ with $y_{0}=1$ and $s_{i}=\sum_{j=0}^{e-1} G\left(\Delta^{j}\right)^{i}$. Since $s_{i} \equiv 0$ $(\bmod p)$ for any $i \geq 1$, we have

$$
y_{i} \equiv 0 \quad(\bmod p) \text { for } 1 \leq i \leq e .
$$

From (2), we see that the numbers $T_{e}(n)$ satisfy the recurrence $\left(p T_{e}(n+e)-p^{n+e}\right)+y_{1}\left(p T_{e}(n+e-1)-p^{n+e-1}\right)+\cdots+y_{e}\left(p T_{e}(n)-p^{n}\right)=0$, that is,

$$
T_{e}(n+e)+y_{1} T_{e}(n+e-1)+\cdots+y_{e} T_{e}(n)-p^{n-1} F_{e}(p)=0 .
$$


Therefore, we have only to find $T_{e}(1), T_{e}(2), \ldots, T_{e}(e)$. It is easy to check that

$$
T_{e}(1)=1, \quad T_{e}(2)= \begin{cases}1+e(p-1) & \text { if }-1 \text { is an } e \text { th power in } \mathbb{F}_{p}, \\ 1 & \text { otherwise }\end{cases}
$$

and

$$
y_{1}=0, \quad y_{2}= \begin{cases}-p e(e-1) / 2 & \text { if }-1 \text { is an } e \text { th power in } \mathbb{F}_{p}, \\ p e / 2 & \text { otherwise }\end{cases}
$$

Next, we will represent $T_{e}(n)$, using the multiplicative character on $\mathbb{F}_{p}^{*}$. From now on, we denote a multiplicative character by $\chi$. We call the character $\chi_{0}$ defined by $\chi_{0}(a)=1$ for all $a \in \mathbb{F}_{p}^{*}$ the trivial character. It is useful to extend the domain of definition of a multiplicative character to all of $\mathbb{F}_{p}$. If $\chi \neq \chi_{0}$ we do this by defining $\chi(0)=0$. For $\chi_{0}$, we define $\chi_{0}(a)=1$ for all $a \in \mathbb{F}_{p}$.

Since

$$
{ }^{\#}\left\{x \in \mathbb{F}_{p} \mid x^{e}=a\right\}=\sum_{\chi^{e}=1} \chi(a)= \begin{cases}e & \text { if } a \text { is an } e \text { th power in } \mathbb{F}_{p}, \\ 1 & \text { if } a=0 \\ 0 & \text { otherwise }\end{cases}
$$

we have

$$
T_{e}(n)=\sum_{\chi_{1}^{e}=1} \sum_{\chi_{2}^{e}=1} \cdots \sum_{\chi_{n}^{e}=1} \sum_{a_{1}+\cdots+a_{n}=0} \chi_{1}\left(a_{1}\right) \chi_{2}\left(a_{2}\right) \cdots \chi_{n}\left(a_{n}\right)
$$

with $a_{1}, \ldots, a_{n} \in \mathbb{F}_{p}$. If $a_{n}=0$, then $\chi_{n}=\chi_{0}$, and the sum is $T_{e}(n-1)$. If $a_{n} \neq 0$, then the sum equals

$$
\begin{aligned}
& \sum_{a_{n}=1}^{p-1} \sum_{\chi_{1}} \cdots \sum_{\chi_{n}} \chi_{1} \cdots \chi_{n-1}(-1) \chi_{1} \cdots \chi_{n}\left(a_{n}\right) \sum_{a_{1}+\cdots+a_{n-1}=1} \chi_{1}\left(a_{1}\right) \cdots \chi_{n-1}\left(a_{n-1}\right) \\
& \quad=(p-1) \sum_{\chi_{1}} \cdots \sum_{\chi_{n-1}} \chi_{1} \cdots \chi_{n-1}(-1) \sum_{a_{1}+\cdots+a_{n-1}=1} \chi_{1}\left(a_{1}\right) \cdots \chi_{n-1}\left(a_{n-1}\right) .
\end{aligned}
$$

Let $S_{e}(n)$ denote

$$
\sum_{\chi_{1}} \cdots \sum_{\chi_{n}} \chi_{1} \cdots \chi_{n}(-1) J_{e}\left(\chi_{1}, \ldots, \chi_{n}\right)
$$

where

$$
J_{e}\left(\chi_{1}, \ldots, \chi_{n}\right)=\sum_{a_{1}+\cdots+a_{n}=1} \chi_{1}\left(a_{1}\right) \cdots \chi_{n}\left(a_{n}\right)
$$

is the generalized Jacobi sum. Hence, we have

$$
T_{e}(n)=T_{e}(n-1)+(p-1) S_{e}(n-1),
$$

and we need to calculate sums of Jacobi sums $S_{e}(2), \ldots, S_{e}(e-1)$. 
Remark. Since $G(\Delta)=\sum_{a}{ }^{\#}\left\{x \in \mathbb{F}_{p} \mid x^{e}=a\right\} \zeta_{p}^{\Delta a}$, from (5) we have $G(\Delta) \equiv 1$ $(\bmod e)$ in $\mathbb{Z}\left[\zeta_{p}\right]$, for $\Delta \not \equiv 0(\bmod p)$. Then $e^{e} \mid F_{e}(p)$.

\section{Properties of generalized Jacobi sums}

In this section, we will study the properties of generalized Jacobi sums. We can calculate them from usual Jacobi sums $J_{e}\left(\chi_{1}, \chi_{2}\right)$ by the following proposition.

Proposition. The generalized Jacobi sum $J_{e}\left(\chi_{1}, \ldots, \chi_{n}\right)$ satisfies the following properties $(\mathrm{a})-(\mathrm{e})$ :

(a) $J_{e}\left(\chi_{1}, \ldots, \chi_{i}, \ldots, \chi_{j}, \ldots, \chi_{n}\right)=J_{e}\left(\chi_{1}, \ldots, \chi_{j}, \ldots, \chi_{i}, \ldots, \chi_{n}\right)$.

(b) If all of the $\chi_{i}(1 \leq i \leq n)$ are trivial, then

$$
J_{e}\left(\chi_{1}, \ldots, \chi_{n}\right)=p^{n-1} .
$$

(c) If some but not all of the $\chi_{i}$ are trivial, then

$$
J_{e}\left(\chi_{1}, \ldots, \chi_{n}\right)=0 \text {. }
$$

(d) If $\chi_{1}, \ldots, \chi_{n}$ and $\chi_{1} \chi_{2}$ are nontrivial for $n \geq 3$, then

$$
J_{e}\left(\chi_{1}, \chi_{2}, \chi_{3}, \ldots, \chi_{n}\right)=J_{e}\left(\chi_{1}, \chi_{2}\right) J_{e}\left(\chi_{1} \chi_{2}, \chi_{3}, \ldots, \chi_{n}\right) \text {. }
$$

(e) If $\chi_{1}, \ldots, \chi_{n}$ are nontrivial and $\chi_{1} \chi_{2}$ is trivial, then

$$
J_{e}\left(\chi_{1}, \chi_{2}, \chi_{3}, \ldots, \chi_{n}\right)=p J_{e}\left(\chi_{3}, \ldots, \chi_{n}\right)
$$

for $n \geq 4$, and $J_{e}\left(\chi_{1}, \chi_{2}, \chi_{3}\right)=p$.

Proof. See [5, pp. 98-101].

\section{THE CASE $e=5$}

For $p$ a prime $\equiv 1(\bmod 5)$, we consider the pair of diophantine equations

$$
\begin{gathered}
16 p=a_{1}^{2}+50 a_{2}^{2}+50 a_{3}^{2}+125 a_{4}^{2}, \\
a_{1} a_{4}=a_{3}^{2}-4 a_{2} a_{3}-a_{2}^{2}, \quad a_{1} \equiv 1 \quad(\bmod 5) .
\end{gathered}
$$

Dickson proved in [4] that this simultaneous system has exactly four solutions, and if one of these is $\left(a_{1}, a_{2}, a_{3}, a_{4}\right)$, the other three are

$$
\left(a_{1},-a_{2},-a_{3}, a_{4}\right),\left(a_{1}, a_{3},-a_{2},-a_{4}\right) \text { and }\left(a_{1},-a_{3}, a_{2},-a_{4}\right) \text {. }
$$

On the other hand, we can choose a prime factor $\pi$ of $p$ in the unique factorization domain $\mathbb{Z}\left[\zeta_{5}\right]$ and a character $\chi$ of order 5 associated with $\pi$, such that

$$
J_{5}(\chi, \chi)=-\pi_{1} \pi_{4},
$$

where $\pi_{i}=\sigma_{i}(\pi)$ and $\sigma_{i}$ is the automorphism of $\mathbb{Q}\left(\zeta_{5}\right)$ defined by $\sigma_{i}\left(\zeta_{5}\right)=$ $\zeta_{5}^{i} \quad(1 \leq i \leq 4)$, and $\zeta_{5}=\exp \left(\frac{2 \pi i}{5}\right)$. If

$$
J_{5}(\chi, \chi)=c_{1} \zeta_{5}+c_{2} \zeta_{5}^{2}+c_{3} \zeta_{5}^{3}+c_{4} \zeta_{5}^{4}
$$


then the four solutions of (8) and (9) correspond to $\sigma_{i}\left(J_{5}(\chi, \chi)\right)=J_{5}\left(\chi^{i}, \chi^{i}\right)$ $(1 \leq i \leq 4)$ by

$$
\begin{aligned}
& 4 c_{1}=-a_{1}+2 a_{2}+4 a_{3}+5 a_{4}, \quad 4 c_{2}=-a_{1}+4 a_{2}-2 a_{3}-5 a_{4}, \\
& 4 c_{3}=-a_{1}-4 a_{2}+2 a_{3}-5 a_{4}, \quad 4 c_{4}=-a_{1}-2 a_{2}-4 a_{3}+5 a_{4} .
\end{aligned}
$$

This result was proved by Dickson in [4].

From properties of Jacobi sums (see [5, 9]):

$$
\begin{aligned}
& J_{e}\left(\chi^{i}, \chi^{j}\right)=J_{e}\left(\chi^{j}, \chi^{i}\right) \\
& =(-1)^{(p-1) j / e} J_{e}\left(\chi^{-i-j}, \chi^{j}\right) \quad(i, j, i+j \not \equiv 0(\bmod p)), \\
& J_{e}\left(\chi^{i}, \chi^{-i}\right)=-\chi(-1)=-1 \quad(i \not \equiv 0 \quad(\bmod p)),
\end{aligned}
$$

we have

$$
\begin{aligned}
J_{5}\left(\chi, \chi^{3}\right)= & J_{5}(\chi, \chi), \quad J_{5}\left(\chi^{4}, \chi^{4}\right)=J_{5}\left(\chi^{2}, \chi^{4}\right)=\sigma_{4}\left(J_{5}(\chi, \chi)\right), \\
& J_{5}\left(\chi^{3}, \chi^{3}\right)=J_{5}\left(\chi^{3}, \chi^{4}\right)=\sigma_{3}\left(J_{5}(\chi, \chi)\right), \\
& J_{5}\left(\chi^{2}, \chi^{2}\right)=J_{5}\left(\chi, \chi^{2}\right)=\sigma_{2}\left(J_{5}(\chi, \chi)\right),
\end{aligned}
$$

and

$$
J_{5}\left(\chi, \chi^{4}\right)=J_{5}\left(\chi^{2}, \chi^{3}\right)=-1 .
$$

Hence, we obtain the following results by direct calculation from the proposition.

Theorem 1. If $\left(a_{1}, a_{2}, a_{3}, a_{4}\right)$ is any solution of (8) and (9), then

$$
\begin{gathered}
S_{5}(2)=p-4+3 a_{1}, \\
S_{5}(3)=p^{2}+36 p+a_{1}{ }^{2}-3 a_{1}-125 a_{4}{ }^{2},
\end{gathered}
$$

and

$$
\begin{aligned}
S_{5}(4)=\frac{1}{8}\left\{a_{1}{ }^{3}-8 a_{1}{ }^{2}-625 a_{1} a_{4}{ }^{2}+392 a_{1} p-2500 a_{2} a_{3} a_{4}\right. \\
\left.+1000 a_{4}{ }^{2}+8 p^{3}-288 p\right\} .
\end{aligned}
$$

From (1), (2), (3), (4) and (7), we can determine the recurrence for $T_{5}(n)$. It is given by

$$
\begin{gathered}
T_{5}(n+5)-10 p T_{5}(n+3)+p Y_{3} T_{5}(n+2)+p Y_{4} T_{5}(n+1) \\
+p Y_{5} T_{5}(n)-5^{5} Z p^{n}=0, \quad n \geq 1,
\end{gathered}
$$

where

$$
Y_{3}=y_{3} / p=-5 a_{1}, \quad Y_{4}=y_{4} / p=-\left(a_{1}^{2}-125 a_{4}^{2}-4 p\right) / 4,
$$

$Y_{5}=y_{5} / p=-\left(a_{1}^{3}-625 a_{1} a_{4}^{2}-8 a_{1} p-2500 a_{2} a_{3} a_{4}\right) / 8$ and $Z=F_{5}(p) / 3125 p$.

For example, for $p<1000$, the values of $a_{1}, a_{2}, a_{3}, a_{4}, Y_{3}, Y_{4}, Y_{5}, Z$ and the first few values are given in Table 1, Table 2 and Table 3. 
TABLE 1

\begin{tabular}{|rrrrr|}
\hline$p$ & $a_{1}$ & $a_{2}$ & $a_{3}$ & $a_{4}$ \\
\hline 11 & 1 & 0 & -1 & 1 \\
31 & 11 & 1 & -2 & 1 \\
41 & -9 & 0 & -3 & -1 \\
61 & 1 & 1 & 4 & -1 \\
71 & -19 & 2 & 3 & 1 \\
101 & -29 & 2 & -3 & -1 \\
131 & 11 & 1 & 6 & 1 \\
151 & -4 & 2 & -2 & -4 \\
181 & 11 & 2 & 7 & -1 \\
191 & 41 & 3 & 4 & -1 \\
211 & 1 & 1 & 2 & -5 \\
241 & 16 & 4 & -4 & 4 \\
251 & -4 & 2 & 6 & 4 \\
271 & 31 & 1 & 8 & 1 \\
281 & 11 & 3 & -4 & 5 \\
311 & -49 & 0 & -7 & -1 \\
331 & 61 & 2 & -5 & 1 \\
401 & -29 & 3 & 10 & 1 \\
421 & -19 & 1 & -8 & -5 \\
431 & 36 & 6 & -6 & 4 \\
461 & 1 & 2 & 9 & 5 \\
491 & -9 & 3 & 12 & 1 \\
521 & 31 & 6 & 7 & -5 \\
541 & -59 & 1 & 10 & -1 \\
571 & -44 & 2 & -10 & -4 \\
601 & -79 & 4 & 7 & 1 \\
631 & -89 & 4 & -5 & -1 \\
641 & 16 & 4 & 12 & -4 \\
661 & 1 & 0 & -3 & 9 \\
691 & 41 & 2 & -11 & 5 \\
701 & -79 & 4 & 9 & 1 \\
751 & 71 & 4 & 11 & -1 \\
761 & -99 & 3 & -6 & -1 \\
811 & 101 & 2 & -7 & 1 \\
821 & 31 & 4 & 15 & -1 \\
881 & 61 & 8 & -9 & 5 \\
911 & 76 & 6 & -10 & 4 \\
941 & -109 & 5 & 6 & 1 \\
971 & -44 & 6 & 14 & 4 \\
991 & -59 & 8 & 11 & 5 \\
\hline & & & &
\end{tabular}

TABLe 2

\begin{tabular}{|rrrrr|}
\hline$p$ & $Y_{3}$ & $Y_{4}$ & $Y_{5}$ & $Z$ \\
\hline 11 & -5 & 210 & 89 & 1 \\
31 & -55 & 160 & 409 & 185 \\
41 & 45 & 260 & -981 & 711 \\
61 & -5 & 460 & -1111 & 3707 \\
71 & 95 & 60 & -101 & 7141 \\
101 & 145 & -390 & -271 & 30463 \\
131 & -55 & 660 & 4009 & 86773 \\
151 & 20 & 3235 & -596 & 155648 \\
181 & -55 & 910 & -1691 & 323951 \\
191 & -205 & -990 & -1331 & 401125 \\
211 & -5 & 4960 & -961 & 604481 \\
241 & -80 & 3385 & 3344 & 1033472 \\
251 & 20 & 3735 & 9004 & 1220224 \\
271 & -155 & 310 & 9599 & 1658645 \\
281 & -55 & 5160 & 5659 & 1923223 \\
311 & 245 & -1290 & -4361 & 2904781 \\
331 & -305 & -2840 & -6541 & 3714113 \\
401 & 145 & 1110 & -1471 & 8075491 \\
421 & 95 & 5560 & -31751 & 9819947 \\
431 & -180 & 3035 & 9684 & 10775808 \\
461 & -5 & 6210 & 30539 & 14139931 \\
491 & 45 & 2510 & 6219 & 18223497 \\
521 & -155 & 5310 & 7349 & 23112547 \\
541 & 295 & -1490 & -13981 & 26932571 \\
571 & 220 & 2935 & -44476 & 33444608 \\
601 & 395 & -4640 & 16729 & 41099279 \\
631 & 445 & -6590 & 31259 & 49981709 \\
641 & -80 & 5385 & -30256 & 53171200 \\
661 & -5 & 15960 & 6989 & 60166491 \\
691 & -205 & 5260 & 65419 & 71870249 \\
701 & 395 & -4140 & 11329 & 76230859 \\
751 & -355 & -2390 & 379 & 100371029 \\
761 & 495 & -8290 & 43839 & 106001415 \\
811 & -505 & -8540 & -43361 & 136615577 \\
821 & -155 & 3060 & 5399 & 143582539 \\
881 & -305 & 3660 & 32009 & 190513423 \\
911 & -380 & -165 & 34364 & 217885696 \\
941 & 545 & -9990 & 60169 & 248389591 \\
971 & 220 & 4935 & 17924 & 281602048 \\
991 & 295 & 4510 & -10531 & 305614349 \\
\hline & & & & \\
\hline
\end{tabular}


TABLE 3

\begin{tabular}{rrrrr}
\hline$p$ & $T_{5}(2)$ & $T_{5}(3)$ & $T_{5}(4)$ & $T_{5}(5)$ \\
\hline 11 & 51 & 151 & 4051 & 19251 \\
31 & 151 & 1951 & 63151 & 1422751 \\
41 & 201 & 601 & 126201 & 2127001 \\
61 & 301 & 3901 & 351301 & 14095501 \\
71 & 351 & 1051 & 553351 & 20697251 \\
101 & 501 & 1501 & 1465501 & 89442501 \\
131 & 651 & 21451 & 2860651 & 303345251 \\
151 & 751 & 21001 & 3960751 & 515455001 \\
181 & 901 & 38701 & 7101901 & 1091506501 \\
191 & 951 & 59851 & 8569951 & 1405510751 \\
211 & 1051 & 45151 & 10333051 & 1984536751 \\
241 & 1201 & 69601 & 15661201 & 3418872001 \\
251 & 1251 & 60001 & 17576251 & 3954325001 \\
271 & 1351 & 98551 & 22762351 & 5504402251 \\
281 & 1401 & 88201 & 24179401 & 6276529001 \\
311 & 1551 & 51151 & 34256551 & 9120099251 \\
331 & 1651 & 169951 & 41383651 & 12338922751 \\
401 & 2001 & 126001 & 70542001 & 25624970001 \\
421 & 2101 & 153301 & 79823101 & 31259728501 \\
431 & 2151 & 232201 & 86432151 & 34836579001 \\
461 & 2301 & 213901 & 104169301 & 45161730501 \\
491 & 2451 & 227851 & 127010451 & 58008735751 \\
521 & 2601 & 319801 & 150048601 & 74096321001 \\
541 & 2701 & 197101 & 170669701 & 84807904501 \\
571 & 2851 & 250801 & 197849851 & 105612051001 \\
601 & 3001 & 219001 & 233733001 & 129031755001 \\
631 & 3151 & 229951 & 270462151 & 156743480251 \\
641 & 3201 & 441601 & 277027201 & 169170752001 \\
661 & 3301 & 438901 & 297828301 & 190917160501 \\
691 & 3451 & 562351 & 346107451 & 228920385751 \\
701 & 3501 & 325501 & 366418501 & 239528747501 \\
751 & 3751 & 723751 & 447528751 & 320096381251 \\
761 & 3801 & 353401 & 468885801 & 332484933001 \\
811 & 4051 & 903151 & 565222051 & 435949431751 \\
821 & 4101 & 750301 & 578309101 & 455370333501 \\
881 & 4401 & 937201 & 712232401 & 604762334001 \\
911 & 4551 & 1037401 & 789338551 & 691887833001 \\
941 & 4701 & 578101 & 876131701 & 779199299501 \\
971 & 4851 & 814801 & 949343851 & 886859651001 \\
991 & 4951 & 806851 & 1008913951 & 961599300751 \\
\hline & & & &
\end{tabular}

5. THE CASE $e=7$

Similarly, for $p$ a prime $\equiv 1(\bmod 7)$, we consider the triple of diophantine equations

$$
\begin{gathered}
72 p=2 a_{1}^{2}+42\left(a_{2}^{2}+a_{3}^{2}+a_{4}^{2}\right)+343\left(a_{5}^{2}+3 a_{6}^{2}\right), \\
12\left(a_{2}^{2}-a_{4}^{2}+2 a_{2} a_{3}-2 a_{2} a_{4}+4 a_{3} a_{4}\right) \\
+49\left(3 a_{5}^{2}+2 a_{5} a_{6}-9 a_{6}^{2}\right)+56 a_{1} a_{6}=0, \\
12\left(a_{2}^{2}-a_{4}^{2}+4 a_{2} a_{3}+2 a_{2} a_{4}+2 a_{3} a_{4}\right) \\
+49\left(a_{5}^{2}+10 a_{5} a_{6}-3 a_{6}^{2}\right)+28 a_{1}\left(a_{5}+a_{6}\right)=0,
\end{gathered}
$$




$$
a_{1} \equiv 1 \quad(\bmod 7) \text {. }
$$

This simultaneous system has six nontrivial solutions in addition to the two trivial solutions $\left(-6 b_{1}, \pm 2 b_{2}, \pm 2 b_{2}, \mp 2 b_{2}, 0,0\right)$, where $b_{1}$ and $b_{2}$ are given by

$$
p=b_{1}^{2}+7 b_{2}^{2}, \quad b_{1} \equiv-1 \quad(\bmod 7) .
$$

If $\left(a_{1}, a_{2}, a_{3}, a_{4}, a_{5}, a_{6}\right)$ is one of the six nontrivial solutions of (12)-(15), the other five nontrivial solutions are

$$
\begin{gathered}
\left(a_{1},-a_{3}, a_{4}, a_{2},-\left(a_{5}+3 a_{6}\right) / 2,\left(a_{5}-a_{6}\right) / 2\right), \\
\left(a_{1},-a_{4}, a_{2},-a_{3},-\left(a_{5}-3 a_{6}\right) / 2,-\left(a_{5}+a_{6}\right) / 2\right), \\
\left(a_{1},-a_{2},-a_{3},-a_{4}, a_{5}, a_{6}\right) \\
\left(a_{1}, a_{3},-a_{4},-a_{2},-\left(a_{5}+3 a_{6}\right) / 2,\left(a_{5}-a_{6}\right) / 2\right), \\
\left(a_{1}, a_{4},-a_{2}, a_{3},-\left(a_{5}-3 a_{6}\right) / 2,-\left(a_{5}+a_{6}\right) / 2\right) .
\end{gathered}
$$

For some character $\chi$ of order 7 , these solutions correspond to Jacobi sums

$$
J_{7}\left(\chi^{i}, \chi^{i}\right)=\sigma_{i}\left(J_{7}(\chi, \chi)\right) \quad(1 \leq i \leq 6),
$$

with

$$
J_{7}(\chi, \chi)=c_{1} \zeta_{7}+c_{2} \zeta_{7}^{2}+c_{3} \zeta_{7}^{3}+c_{4} \zeta_{7}^{4}+c_{5} \zeta_{7}^{5}+c_{6} \zeta_{7}^{6},
$$

where

$$
\begin{array}{ll}
12 c_{1}=-2 a_{1}+6 a_{2}+7 a_{5}+21 a_{6}, & 12 c_{2}=-2 a_{1}+6 a_{3}+7 a_{5}-21 a_{6}, \\
12 c_{3}=-2 a_{1}+6 a_{4}-14 a_{5}, & 12 c_{4}=-2 a_{1}-6 a_{4}-14 a_{5}, \\
12 c_{5}=-2 a_{1}-6 a_{3}+7 a_{5}-21 a_{6}, & 12 c_{6}=-2 a_{1}-6 a_{2}+7 a_{5}+21 a_{6},
\end{array}
$$

and $\sigma_{i}$ is the automorphism of $\mathbb{Q}\left(\zeta_{7}\right)$ defined by $\sigma_{i}\left(\zeta_{7}\right)=\zeta_{7}^{i} \quad(1 \leq i \leq 6)$. The trivial solutions correspond to Jacobi sums $\sigma_{i}\left(J_{7}\left(\chi, \chi^{2}\right)\right)(i=1,6)$ with

$$
J_{7}\left(\chi, \chi^{2}\right)=b_{1}+b_{2} \sqrt{-7} .
$$

These results were proved by Leonard and Williams in [6]. Since

$$
\begin{gathered}
J_{7}\left(\chi, \chi^{5}\right)=J_{7}(\chi, \chi), \quad J_{7}\left(\chi^{6}, \chi^{6}\right)=J_{7}\left(\chi^{2}, \chi^{6}\right), \quad J_{7}\left(\chi^{2}, \chi^{2}\right)=J_{7}\left(\chi^{2}, \chi^{3}\right), \\
J_{7}\left(\chi^{5}, \chi^{5}\right)=J_{7}\left(\chi^{4}, \chi^{5}\right), \quad J_{7}\left(\chi^{3}, \chi^{3}\right)=J_{7}\left(\chi, \chi^{3}\right), \\
J_{7}\left(\chi^{4}, \chi^{4}\right)=J_{7}\left(\chi^{4}, \chi^{6}\right), \quad J_{7}\left(\chi, \chi^{4}\right)=J_{7}\left(\chi^{2}, \chi^{4}\right)=J_{7}\left(\chi, \chi^{2}\right), \\
J_{7}\left(\chi^{3}, \chi^{5}\right)=J_{7}\left(\chi^{3}, \chi^{6}\right)=J_{7}\left(\chi^{5}, \chi^{6}\right)=\sigma_{6}\left(J_{7}\left(\chi, \chi^{2}\right)\right), \quad \text { and } \\
J_{7}\left(\chi, \chi^{6}\right)=J_{7}\left(\chi^{2}, \chi^{5}\right)=J_{7}\left(\chi^{3}, \chi^{4}\right)=-1,
\end{gathered}
$$

from (10) and (11) we obtain the following results from the proposition.

Theorem 2. If $\left(a_{1}, a_{2}, a_{3}, a_{4}, a_{5}, a_{6}\right)$ is any nontrivial solution of (12)-(15) with $a_{1} \equiv 1(\bmod 7)$ and $\left(b_{1}, b_{2}\right)$ is the solution of $(16)$ with $b_{1} \equiv-1(\bmod 7)$, then

$$
\begin{gathered}
S_{7}(2)=p-6+3 a_{1}+12 b_{1}, \\
S_{7}(3)=p^{2}+p-6-S_{7}(2)+66 p+2 a_{1}^{2}+8 a_{1} b_{1}+14 U_{1}-28 U_{2},
\end{gathered}
$$




$$
\begin{gathered}
S_{7}(4)=\frac{p^{4}-1}{p-1}-7-S_{7}(2)-S_{7}(3) \\
+\frac{5}{144}\left\{8 a_{1}^{3}+144 a_{1}^{2} b_{1}+2736 a_{1} p-168 a_{1} U_{2}\right. \\
+8640 b_{1} p+1008 b_{1} U_{1} \\
\left.\quad-2016 b_{1} U_{2}-4116 V_{1}(0,1)+2401 V_{2}(5,-9)\right\} \\
\begin{array}{r}
S_{7}(5)=\frac{p^{5}-1}{p-1}-7-S_{7}(2)-S_{7}(3)-S_{7}(4) \\
+\frac{1}{12}\left\{8 a_{1}^{3} b_{1}+972 a_{1}^{2} p+4896 a_{1} b_{1} p-168 a_{1} b_{1} U_{2}+4320 b_{1}^{2} p\right. \\
-4116 b_{1} V_{1}(0,1)+2401 b_{1} V_{2}(5,-9)+9576 p^{2} \\
\left.+5544 p U_{1}-9072 p U_{2}\right\}
\end{array}
\end{gathered}
$$

and

$$
\begin{aligned}
S_{7}(6)=\frac{p^{6}-1}{p-1} & -7-S_{7}(2)-S_{7}(3)-S_{7}(4)-S_{7}(5) \\
-\frac{1}{864}\{ & 34 a_{1}^{4} b_{1}-5688 a_{1}^{3} p-334368 a_{1}^{2} b_{1} p+1512 a_{1}^{2} b_{1} U_{1} \\
& +504 a_{1}^{2} b_{1} U_{2}-362880 a_{1} b_{1}^{2} p+24696 a_{1} b_{1} V_{1}(0,1) \\
& -4802 a_{1} b_{1} V_{2}(13,27)-2334528 a_{1} p^{2}+77112 a_{1} p U_{1} \\
& +99792 a_{1} p U_{2}-5145120 b_{1} p^{2}-1829520 b_{1} p U_{1} \\
& +2116800 b_{1} p U_{2}+2646 b_{1} U_{1}^{2}-3087 b_{1} W_{1}(-2,15,27) \\
& -6174 b_{1} W_{2}(4,12,9)-10584 b_{1} W_{3}+3630312 p V_{1}(0,1) \\
& -24696 p V_{1}(1,0)-1210104 p V_{1}(1,-1) \\
& +14406 p V_{2}(11,-45)-2117682 p V_{2}(5,-9) \\
& \left.-1411788 p V_{2}(1,36)\right\},
\end{aligned}
$$

where

$$
\begin{gathered}
U_{1}=a_{2}^{2}+a_{3}^{2}+a_{4}^{2}, \quad U_{2}=a_{2} a_{3}-a_{2} a_{4}-a_{3} a_{4}, \\
V_{1}(i, j)=(i-j) a_{2} a_{3} a_{5}+(2 i+j) a_{2} a_{4} a_{5}-(i+2 j) a_{3} a_{4} a_{5} \\
+3(i+j) a_{2} a_{3} a_{6}+3 j a_{2} a_{4} a_{6}+3 i a_{3} a_{4} a_{6}, \\
V_{2}(i, j)=i a_{5}^{3}+j a_{5}^{2} a_{6}-9 i a_{5} a_{6}^{2}-j a_{6}^{3}, \\
W_{1}(i, j, k)=(i-j+k) a_{2}^{2} a_{5}^{2}+2(3 i-j-k) a_{2}^{2} a_{5} a_{6}+(9 i+3 j+k) a_{2}^{2} a_{6}^{2} \\
+(i+j+k) a_{3}^{2} a_{5}^{2}-2(3 i+j-k) a_{3}^{2} a_{5} a_{6}+(9 i-3 j+k) a_{3}^{2} a_{6}^{2} \\
+4 i a_{4}^{2} a_{5}^{2}+4 j a_{4}^{2} a_{5} a_{6}+4 k a_{4}^{2} a_{6}^{2}, \\
W_{2}(i, j, k)=-(i-j+k) a_{2} a_{3} a_{5}^{2}-2(3 i-j-k) a_{2} a_{3} a_{5} a_{6} \\
-(9 i+3 j+k) a_{2} a_{3} a_{6}^{2}+(i+j+k) a_{3} a_{4} a_{5}^{2} \\
-2(3 i+j-k) a_{3} a_{4} a_{5} a_{6}+(9 i-3 j+k) a_{3} a_{4} a_{6}^{2} \\
+4 i a_{2} a_{4} a_{5}^{2}+4 j a_{2} a_{4} a_{5} a_{6}+4 k a_{2} a_{4} a_{6}^{2}, \\
W_{3}=a_{2}^{3} a_{3}-a_{3}^{3} a_{4}-a_{4}^{3} a_{2} .
\end{gathered}
$$


Note, that $U_{1}, \ldots, W_{3}$ are invariants under the change of the solution of (12)-(15).

It is hard to check the results of this theorem, but we calculated them as follows. Let $\mathscr{G}_{i}=\sigma_{i}\left(J_{7}(\chi, \chi)\right) \quad(1 \leq i \leq 6)$ and $\mathscr{H}_{i}=\sigma_{i}\left(J_{7}\left(\chi, \chi^{2}\right) \quad(i=\right.$ $1,6)$. First, we expressed $S_{7}(2), \ldots, S_{7}(6)$ in terms of 10 polynomials:

$$
\begin{gathered}
\mathscr{I}_{1}+\mathscr{G}_{2}+\mathscr{G}_{3}+\mathscr{G}_{4}+\mathscr{G}_{5}+\mathscr{G}_{6}, \\
\mathscr{G}_{1}^{2}+\mathscr{G}_{2}^{2}+\mathscr{G}_{3}^{2}+\mathscr{G}_{4}^{2}+\mathscr{G}_{5}^{2}+\mathscr{G}_{6}^{2}, \\
\mathscr{H}_{1}+\mathscr{H}_{2}, \quad \mathscr{H}_{1}^{2}+\mathscr{H}_{2}^{2}, \\
\mathscr{G}_{1} \mathscr{G}_{2}+\mathscr{G}_{2} \mathscr{G}_{4}+\mathscr{G}_{4} \mathscr{G}_{1}+\mathscr{G}_{3} \mathscr{G}_{5}+\mathscr{G}_{5} \mathscr{G}_{6}+\mathscr{G}_{6} \mathscr{G}_{3}, \\
\mathscr{G}_{1} \mathscr{G}_{3}+\mathscr{G}_{1} \mathscr{G}_{5}+\mathscr{G}_{2} \mathscr{G}_{3}+\mathscr{G}_{2} \mathscr{G}_{6}+\mathscr{G}_{4} \mathscr{G}_{5}+\mathscr{G}_{4} \mathscr{G}_{6}, \\
\mathscr{G}_{1}^{2} \mathscr{G}_{2}+\mathscr{G}_{2}^{2} \mathscr{G}_{4}+\mathscr{G}_{4}^{2} \mathscr{G}_{1}+\mathscr{G}_{3} \mathscr{G}_{5}^{2}+\mathscr{G}_{5} \mathscr{G}_{6}^{2}+\mathscr{G}_{6} \mathscr{G}_{3}^{2}, \\
\mathscr{G}_{1}^{2} \mathscr{G}_{3}+\mathscr{G}_{1} \mathscr{G}_{5}^{2}+\mathscr{G}_{2}^{2} \mathscr{G}_{3}+\mathscr{G}_{2} \mathscr{G}_{6}^{2}+\mathscr{G}_{4}^{2} \mathscr{G}_{5}+\mathscr{G}_{4} \mathscr{G}_{6}^{2}, \\
\mathscr{G}_{1}^{2} \mathscr{H}_{2}+\mathscr{G}_{2}^{2} \mathscr{H}_{2}+\mathscr{G}_{3}^{2} \mathscr{H}_{1}+\mathscr{G}_{4}^{2} \mathscr{H}_{2}+\mathscr{G}_{5}^{2} \mathscr{H}_{1}+\mathscr{G}_{6}^{2} \mathscr{H}_{1}, \\
\mathscr{G}_{1}^{3} \mathscr{G}_{2}+\mathscr{G}_{2}^{3} \mathscr{G}_{4}+\mathscr{G}_{4}^{3} \mathscr{G}_{1}+\mathscr{G}_{3} \mathscr{G}_{5}^{3}+\mathscr{G}_{5} \mathscr{G}_{6}^{3}+\mathscr{G}_{6} \mathscr{G}_{3}^{3} .
\end{gathered}
$$

Next, substituting (17) and (18) for them, they may be expressed as a polynomial in $p, a_{1}, b_{1}, U_{1}, \ldots, W_{3}$.

Notice that $b_{2}$ is not needed in Theorem 2 , but is determined by

$$
J_{7}(\chi, \chi) J_{7}\left(\chi^{2}, \chi^{2}\right) J_{7}\left(\chi^{4}, \chi^{4}\right)=p J_{7}\left(\chi, \chi^{2}\right) .
$$

The recurrence for $T_{7}(n)$ is given by

$$
\begin{aligned}
T_{7}(n+7) & -21 p T_{7}(n+5)+p Y_{3} T_{7}(n+4)+p Y_{4} T_{7}(n+3)+p Y_{5} T_{7}(n+2) \\
& +p Y_{6} T_{7}(n+1)+p Y_{7} T_{7}(n)-7^{7} Z p^{n}=0,
\end{aligned}
$$

where

$$
\begin{aligned}
Y_{3}=y_{3} / p= & \frac{7}{3}\left(p-6-S_{7}(2)\right), \\
Y_{4}=y_{4} / p= & \frac{7}{4}\left(p^{2}+127 p-6-S_{7}(2)-S_{7}(3)\right), \\
Y_{5}=y_{5} / p= & \frac{7}{5}\left(p^{3}-34 p^{2}+35 p S_{7}(2)+211 p-6-S_{7}(2)-S_{7}(3)-S_{7}(4)\right), \\
Y_{6}=y_{6} / p=\frac{7}{36}\left(6 p^{4}-169 p^{3}-28 p^{2} S_{7}(2)-8289 p^{2}+14 p S_{7}(2)^{2}+357 p S_{7}(2)\right. & \\
& \left.+189 p S_{7}(3)+1644 p-36\right)-\frac{7}{6}\left(S_{7}(2)+S_{7}(3)+S_{7}(4)+S_{7}(5)\right), \\
Y_{7}=y_{7} / p=\frac{1}{60} & \left(60 p^{5}-1459 p^{4}-245 p^{3} S_{7}(2)+27941 p^{3}\right. \\
& \quad 31360 p^{2} S_{7}(2)-245 p^{2} S_{7}(3)-189864 p^{2}+245 p S_{7}(2)^{2} \\
& +245 p S_{7}(2) S_{7}(3)+4704 p S_{7}(2)+3234 p S_{7}(3)+1764 p S_{7}(4) \\
& +19464 p-360)-\left(S_{7}(2)+S_{7}(3)+S_{7}(4)+S_{7}(5)+S_{7}(6)\right), \\
Z=F_{7}(p) / 823543 p . &
\end{aligned}
$$

For example, for $p<1000$, we have Table 4, Table 5 and Table 6 . 
TABLE 4

\begin{tabular}{rrrrrrrr}
\hline$p$ & $a_{1}$ & $a_{2}$ & \multicolumn{1}{c}{$a_{3}$} & $a_{4}$ & \multicolumn{1}{c}{$a_{5}$} & $a_{6}$ & $b_{1}$ \\
\hline 29 & 1 & 2 & 3 & 2 & -1 & 1 & -1 \\
43 & 1 & 6 & 1 & 2 & -1 & 1 & 6 \\
71 & 15 & 2 & 0 & -3 & 0 & 2 & -8 \\
113 & -27 & 3 & -6 & -4 & -3 & 1 & -1 \\
127 & 29 & 1 & 0 & -12 & 1 & 1 & -8 \\
197 & -13 & 6 & -1 & 8 & -5 & 1 & 13 \\
211 & -55 & 13 & 4 & 0 & 1 & 1 & 6 \\
239 & 57 & 0 & 6 & -11 & 0 & 2 & -8 \\
281 & 57 & 12 & -6 & 7 & 0 & 2 & 13 \\
337 & -13 & 4 & -15 & -10 & 1 & 3 & -15 \\
379 & -13 & 10 & 13 & -12 & -5 & 1 & 6 \\
421 & -55 & 4 & -3 & -18 & -5 & 1 & 13 \\
449 & -41 & 19 & 0 & 10 & 5 & 1 & -1 \\
463 & 1 & 9 & -22 & 0 & 5 & 1 & 20 \\
491 & -69 & 9 & -20 & -6 & -3 & 1 & -22 \\
547 & 43 & 2 & -15 & 0 & -1 & 5 & -22 \\
617 & -55 & 16 & -6 & 1 & -8 & 2 & 13 \\
631 & 8 & 14 & 6 & -18 & 4 & 4 & -8 \\
659 & -27 & 9 & -30 & -4 & -3 & 1 & -22 \\
673 & 22 & 12 & 30 & -8 & -4 & 4 & -15 \\
701 & -125 & 20 & 3 & -4 & -1 & 1 & -1 \\
743 & -27 & 3 & 20 & -12 & 9 & 1 & 20 \\
757 & -27 & 13 & 4 & 14 & -9 & 3 & 27 \\
827 & 15 & 3 & 6 & -26 & 9 & 1 & -22 \\
883 & 15 & 13 & -32 & -4 & 3 & 3 & 6 \\
911 & 29 & 6 & 10 & 31 & -2 & 4 & -8 \\
953 & 50 & 12 & 8 & -28 & 4 & 4 & -29 \\
967 & 127 & 15 & -6 & 20 & -1 & 3 & 20 \\
\hline
\end{tabular}


TABLE 5

\begin{tabular}{rrrrrrr}
\hline$p$ & $Y_{3}$ & $Y_{4}$ & $Y_{5}$ & $Y_{6}$ & $Y_{7}$ & $Z$ \\
\hline 29 & 21 & 2443 & 868 & -45556 & 70643 & 289 \\
43 & -175 & 3031 & 32816 & -54768 & -919339 & 3563 \\
71 & 119 & 8323 & -83706 & -263900 & 1928275 & 116297 \\
113 & 217 & 5971 & -20790 & -28112 & 35447 & 2111327 \\
127 & 21 & 10675 & -36176 & -1546524 & 8841251 & 4284689 \\
197 & -273 & 17731 & 288890 & -1485568 & -3682589 & 63088549 \\
211 & 217 & 14203 & -33824 & -2902452 & -15278033 & 97171097 \\
239 & -175 & 19495 & 11550 & -4478096 & 22306759 & 206053139 \\
281 & -763 & -3437 & 132090 & 947968 & -2081087 & 547263017 \\
337 & 511 & 15379 & -588014 & 4909128 & -7359899 & 1676462987 \\
379 & -77 & 50071 & 396886 & -34381032 & -386244419 & 3400759571 \\
421 & 21 & 35371 & 356902 & -7864584 & -88796149 & 6427762907 \\
449 & 315 & 20083 & 92890 & -268408 & -1427749 & 9501724321 \\
463 & -567 & 24787 & 676382 & -6245820 & -95682959 & 11390859329 \\
491 & 1099 & -11081 & -265398 & 2764888 & 30094973 & 16362064007 \\
547 & 315 & 57127 & -1490398 & -9087624 & 118738969 & 31322841539 \\
617 & 21 & 51835 & 350042 & -14093464 & 59902093 & 64730776477 \\
631 & 168 & 75061 & -1075760 & -68095335 & 1009965704 & 74149915136 \\
659 & 805 & 16555 & -820470 & -1070636 & 145798841 & 96475302113 \\
673 & 266 & 80549 & -1209824 & -128631013 & 8403667999 & 109399460603 \\
701 & 903 & 14203 & -618296 & -8719732 & -12731797 & 140039937427 \\
743 & -371 & 85939 & 2575524 & -72044588 & -2482867129 & 198422927777 \\
757 & -567 & 68691 & 2678130 & 190232 & -61712631 & 221974547877 \\
827 & 511 & 85351 & -2525964 & -97022240 & 3008326775 & 378942918455 \\
883 & -273 & 36939 & -292152 & -8141140 & 93385725 & 561684402621 \\
911 & 21 & 47719 & 426188 & -16060912 & -188652563 & 678164698651 \\
953 & 462 & 119455 & -3968524 & -190379539 & 7358806846 & 890182377472 \\
967 & -1449 & -19901 & 636986 & 19188708 & 135683491 & 969704283077 \\
\hline & & & & & &
\end{tabular}


TABLE 6

\begin{tabular}{rrrrrrr}
\hline$p$ & $T_{7}(2)$ & $T_{7}(3)$ & $T_{7}(4)$ & $T_{7}(5)$ & $T_{7}(6)$ & $T_{7}(7)$ \\
\hline 29 & 197 & 589 & 87613 & 434141 & 48359081 & 401624189 \\
43 & 295 & 4999 & 234319 & 7175071 & 279635161 & 10150704271 \\
71 & 491 & 1471 & 651211 & 20725531 & 2039344721 & 123105907051 \\
113 & 785 & 2353 & 2655409 & 123515281 & 21106338401 & 1967012774161 \\
127 & 883 & 14995 & 3296035 & 258359851 & 35510978161 & 4191682116511 \\
197 & 1373 & 61741 & 10524613 & 1623810021 & 305995801721 & 58947076303389 \\
211 & 1471 & 24991 & 13272631 & 1842963991 & 433054373641 & 87522476780971 \\
239 & 1667 & 74971 & 18167731 & 3410160391 & 797482745921 & 187210905127219 \\
281 & 1961 & 170521 & 32651641 & 7108914121 & 1834761374321 & 499737158893561 \\
337 & 2353 & 39985 & 49587217 & 12171117841 & 4427480228641 & 1458494959685905 \\
379 & 2647 & 156115 & 61675615 & 20691045271 & 7845859876681 & 2963950323867163 \\
421 & 2941 & 173461 & 88408741 & 31251603181 & 13312709379481 & 5566068700912861 \\
449 & 3137 & 141121 & 110722753 & 39662795201 & 18423611731841 & 8181996374934593 \\
463 & 3235 & 326635 & 119661235 & 47550132631 & 21475297314241 & 9869891508002527 \\
491 & 3431 & 10291 & 151787791 & 54246811711 & 29020877711561 & 13952783047781971 \\
547 & 3823 & 225499 & 183475111 & 88696102951 & 49112851733401 & 26778955559333251 \\
617 & 4313 & 375145 & 264528265 & 144650373561 & 89691572093201 & 55166427521113833 \\
631 & 4411 & 352801 & 274306411 & 158014498321 & 100202011231771 & 63116105003804161 \\
659 & 4607 & 207271 & 334602871 & 183749622211 & 125035320648281 & 81817099907716363 \\
673 & 4705 & 376321 & 330874657 & 203920893121 & 138299815325281 & 92895803047928161 \\
701 & 4901 & 220501 & 400619101 & 235137559301 & 170235370769801 & 118536720987233501 \\
743 & 5195 & 670027 & 443198827 & 306461088571 & 226744647572801 & 168262868716824967 \\
757 & 5293 & 756757 & 476232373 & 331806309661 & 249105000979801 & 188234683135268869 \\
827 & 5783 & 503035 & 611394463 & 464013218971 & 387427058908793 & 319850098013903395 \\
883 & 6175 & 882883 & 767977687 & 611288203051 & 538121706358825 & 474064223649480703 \\
911 & 6371 & 821731 & 835699411 & 688230705891 & 628767383411441 & 571610334899603091 \\
953 & 6665 & 719713 & 914853913 & 821254899921 & 786651279340361 & 749072885603310465 \\
967 & 6763 & 1534975 & 1032915787 & 894255059251 & 849085922746321 & 818225339860370275 \\
\hline & & & & & & \\
\end{tabular}




\section{ACKNOWLEDGMENT}

The author is grateful to Professor Y. Mimura and the referee for many suggestions and comments.

\section{BIBLIOGRAPHY}

1. L.D. Baumert and H. Fredricksen, The cyclotomic numbers of order eighteen with applications to difference sets, Math. Comp. 21 (1967), 204-219.

2. B.C. Berndt and R.J. Evans, Sums of Gauss, Jacobi, and Jacobsthal, J.Number Theory 11 (1979), 349-398.

3. S. Chowla, J. Cowles and M. Cowles, On the number of zeros of diagonal cubic forms, J. Number Theory 9 (1977), 502-506.

4. L.E. Dickson, Cyclotomy higher congruences, and Waring's problem I,II, Amer. J. Math. 57 (1935), 463-474.

5. K. Ireland and M. Rosen, A classical introduction to modern number theory, Graduate Texts in Math., vol. 84, Springer-Verlag, New York, Heidelberg, Berlin, 1982.

6. P.A. Leonard and K.S. Williams, The cyclotomic numbers of order seven, Proc. Amer. Math. Soc. 51 (1975), 295-300.

7. __ The cyclotomic numbers of order eleven, Acta Arith. 26 (1975), 366-383.

8. J.B. Muskat, The cyclotomic numbers of order fourteen, Acta Arith. 11 (1966), 263-279.

9. __ On Jacobi sums of certain composite order, Trans. Amer. Math. Soc. 134 (1969), 483-502.

10. J.B. Muskat and A.L. Whiteman, The cyclotomic numbers of order twenty, Acta Arith. 17 (1970), 185-216.

11. G. Myerson, On the number of zeros of diagonal cubic forms, J. Number Theory 11 (1979), 95-99.

12. D.R. Richman, Some remarks on the number of solutions to the equation $f\left(X_{1}\right)+\cdots+f\left(X_{n}\right)$ = 0, Stud. Appl. Math. 71 (1984), 263-266.

13. A.L. Whiteman, The cyclotomic numbers of order sixteen, Trans. Amer. Math. Soc. 86 (1957), 401-413.

14. _ The cyclotomic numbers of order ten, Proc. Sympos. Appl. Math., vol. 10, Amer. Math. Soc., Providence, RI, 1960, pp. 95-111.

Department of Mathematics, Osaka Institute of Technology, Ohmiya, Asahi-ku, OSAKA 535, JAPAN 